\title{
Discovery, invention and serendipity
}

\author{
Akira Ishikawa ${ }^{1,2}$ \\ (1. Aoyama Gakuin University, Tokyo 107-0062, Japan; 2. ICC Institute, University of Texas at Austin, Austin 78712, USA)
}

\begin{abstract}
This paper attempts to relate serendipity, the natural ability to make interesting or valuable discoveries by accident (Longman Discovery of Contemporary English) with notable discoveries and inventions. It connotes the profound ability of finding out valuable things different from those who have been exploring by spending a lot of time or for years. The author will illustrate as many cases as possible within a given time constraint, so that surrounding environments and situations may be clarified, and such discoveries and inventions may be plausibly accomplished by accident or with rich reasons. These illustrations include historical as well as more recent cases. One of the cases is beyond the scope of our environment on the earth with preconceived notion, while other cases have given great impacts to our politics and also military intelligence. It should be noted that business and technological intelligence are deeply connected with discoveries, inventions and serendipity. In concluding remarks, the essence of serendipity will be summarized and how to side with the serendipity at the most important moment will be examined by viewing time series endeavors of the inventors and those who have devoted much time to discoveries.
\end{abstract}

Key words: discovery; invention; serendipity

\section{Introduction}

First of all, two examples of happenstance, mistake and successive discoveries will be illustrated.

In order to create artificial snow, Dr. Ukichirou Nakaya failed over and over again before finally succeeding in creating an artificial snow crystal through the use of a Japanese hare. While this discovery was truly unexpected, an examination of the hair of the hare through a microscope revealed strands of stinging hair growing in many layers. It then turned out to be clear that their structure was exactly fit for creating crystals of artificial snow. There was no such an object found before.

In this example, the very hair of the hare was the directly sought-after information source by observation unexpected, but indirectly, the source was an element of the natural environment found outside of the research facility free to be observed by anyone. In this sense, happenstance and unexpectedness have sided with this great discovery.

Another famous example is the case of Dr. Alexander Fleming, who has sprinkled a bacteria called staphylococcus on a petridish and left it there without sealing by mistake. Then by chance, some green mould had fallen into the dish. Consequently, although it is completely unexpected, he ended up with observing the staphylococcus melting away, and it is said that it is very this observation that led him to the discovery of penicillin.

In this case, mistake resulted in opening his eyes. And his successive observation succeeded in finding out

\footnotetext{
Akira Ishikawa, Ph.D., professor emeritus, Aoyama Gakuin University, and senior research fellow, ICC Institute, University of Texas at Austin; research fields: new product and new business development, crisis and risk management, knowledge management, system sciences, business intelligence, gloval innovation, intellectual development and Olympics, fuzzy systems, strategic budgeting.
} 
the utterly unexpected phenomenon. One of the keen differences from the former case is whether or not the cognition of mistake has been made in advance.

\section{Mistakes, judgment and discovery}

The third case is similar to the previous case in that the cognition of mistake existed. However, audacious judgment and decision has led to the discovery rather than successive observation only.

Mr. Koichi Tanaka won the Nobel Prize for Chemistry in 2002. Mr. Tanaka was absorbed in his work on the mass spectrometry of the protein. In this case, it was necessary to vaporize and ionize the protein, but while the protein is a substance that is difficult to vaporize on the one hand, to ionize it, a high level of energy is required, on the other.

However, since applying a high level of energy fails to vaporize the protein and only leads to its decomposition, it had been extremely difficult to ionize something with a particularly high-molecular weight as protein.

He then accidentally went on to mix glycerol and cobalt by mistake and upon attempting to use the mixture as a thermal energy shock absorber, since he did not wish to see it go to waste, he unexpectedly became the first person in the world to completely successful such an attempt. This method was named the "soft laser ion method" and it went on to be awarded the Nobel Prize for Chemistry for its achievements.

The fourth illustration is a drag named Aricept which is known as donepezil developed by a specialist at Eisai. Aricept was a drag developed for the treatment of Alzheimer's disease. It received a special commendation in the 1997 UK Prix Galian Awards, considered as the equivalent of the Nobel Prize for medical research.

In the process of development and discovery, a group of specialists called attention to the Choline hypothesis in that the more the density in the brain of a neurotransmitter gets higher, the more the memory block is improved. This hypothesis was falling into obscurity at that time, with a belief that it was ineffective.

Nevertheless, it drew the attention of the Eisai specialists, and eventually paved the way towards its development into a drug whose sales went on to exceed 100 billion yen in Japan alone in 2002.

This case is a challenge against a preconceived notion of disbelief and misjudgment. It also instructs us to re-examine what has been disbelieved as a truth. A truth behind truth hints us to explore the matter.

The fifth case is somewhat similar to the fourth one. Dr. Reona Esaki discovered an effect of electron tunneling which exists where metallic and magnetic properties intersect. This discovery is, in fact, the harbinger of the transistor age. The phenomenon, however, was the apparently improbable appearance of electrons on the both side of a compositional surface in a manner that suggests that they have passed through a tunnel.

He had announced it to the domestic Physical Society, but the reaction of the scientists was extremely indifferent, as it was seen to apparently violate the theories of classical physics at that time.

However, it is known that the discovery began to attract attention once Dr. Esaki received acclaim from Dr. William Shockley, a pioneer in transistor research, and eventually, it was commercialized, after Dr. Esaki announced the discovery at an international convention in Brussels upon publishing an article on the discovery in an American journal in 1958. Because of this accomplishment, he later awarded the Nobel Prize.

\section{Ignorance, judgment and discovery}

Dr. Sumio Iijima who discovered Carbon Nanotube seems to worth introducing as the sixth example, because this is a noteworthy example in which wastes became valuables in 1991. Carbon Nanotube is a new 
carbon substance after the discovery of Diamond, Graphite and Fullerene.

He says that a discovering power of humans by accident or unexpected has been observed in numerous examples, including the principle of Archimedis, discovery of Pluto, discoveries of Drs. Koshiba and Nozoe lead to the Nobel Prize.

In fact, Professor Iijima's discovery is of serendipity. He happened to notice piles of electrodes (poles) finished for producing fullerenes in his colleague's laboratory in Meijo University at the time when studies on fullerene were so prevalent. It was nothing but wastes. Due to his extraordinary curiosity, he observed the structure of this fullerene through an electronic microscope and found out a lengthy carbon structure which he never expected.

The seventh case is related to the development of a binding agent by Art Fly. By accident, he developed one that is easily peeled off by mistake. He, however, used it for the bookmark of his hymnbook after coating the thin paper with this incomplete binding agent. This is the origin of the so popular Post-It.

This case implies that we should never give up so easily even if we have made a mistake. Alternate uses might always be hidden. Whether or not we may discover is our own insight from different angles and thoughts.

The eighth case is on the development of Biagra. This medicine was originally developed for the purpose of curing the heart disease. However, the developers happened to notice its side effect, because the patients who joined their clinical studies never wanted to return the drug, even if it is not specifically effective for their heart diseases.

The ninth and final example in this section is connected with a radar system in the World War II. An Instructor called Stanley Hay was dispatched to the Operations Research Group in the army to join the Radar Research Project in Great Britain. While he was ordered to check the condition of the receipt and transmission of electronic wave, he was also directed to explore strange phenomena that was taken place on the radar.

It was an on-off switching effect like a Christmas tree often happens, which had made them difficult to discern enemy bombers. They considered that as a result of developing a new radar electro-wave block technology, strong anti-electronic wave must have been transmitted to the radar base in England.

He noticed that the waves come from east in the morning, from south at noon and west in the afternoon and disappear after dark, and began to explore an effective countermeasure approach by finding out the feature of them. In Spring, 1942, they concluded that this phenomenon has nothing to do with the blocking wave from Germany.

According to his investigation, this wave did not come from Germany, but from sun. Incidentally, sun was at the peak of the sunspot in its eleventh year cycle. Thus, he discovered that the wave had something to do with the sunspot and that every sunspot transmitted respective waves. This discovery enabled the inference that every star transmits a kind of waves. Later, Hey further discovered that meteorites also send electronic signal when they enter into the atmospheric zone, while he maneuvered a special radar that investigates a wide-angle to observe the coming V-2 rocket.

\section{Preconceived notion, judgment and discovery}

The tenth case is concerned with what is called preconceived notion. It is very difficult to surmount our preconceived notion, even if we try to overcome.

In 1970, Russia sent Moon surface explorer, Luna 16, to collect samples of soil on the surface of moon and also to transmit moon surface images to the earth. More than two years before shooting, an electric bulb for this explorer had been developed. However, as a result of simulations repeated many times, glasses on the surface of 
the electric bulb could not stand with the physical impact of moon surface landing and was cracked so easily.

At this point, an engineer who was well versed in TRIZ, an innovative approach for problem-solving, proposed a non-glass bulb. While a glass is indispensable on the earth, the important function of glasses which is to make vacuous has been completely eliminated, since there is no oxygen on the moon. We have been inflicted with a preconceived notion that different environment is the same with our world.

As a result, this electric bulb issues have been easily resolved after spending much time and efforts with less costs involved.

The eleventh and final illustration this time is the case of escaping from the physical risk. Husband of a professor at Kentucky State University had been suffered from diabetes for years. To make the matters worse, he was also afflicted with osteomyelitis. As a result of examination by a physician, amputation from his knee was his decision. Upon hearing the advice, the professor disbelieved in this treatment and decided to find out alternative treatments.

She utilized Text Miner that is one of the Business Intelligence Tools and was sold by SAS, one of the vendors. The similar case has been reported from Nagasaki Medical Center to predict the fatality of cancer patients. Consequently, she fortunately found an alternative method so that the husband may not have to amputate his body. This case implies that among many alternatives, it is not impossible to discover an alternative out of employing data warehouses available.

\section{Concluding remarks}

Eleven cases have been illustrated with emphasis on serendipity in the process of discovery and invention. They imply that unexpectedness and happenstance took place under different environments, different methods and tools and different psychological situation.

While it is extremely difficult to find what is unexpected, it is more likely that we can approach by abandoning our preconceived notion, by not easily discarding the experiments and trials we failed, and by continuously making efforts steadily so that we may gradually beckon the angel of unexpected fortune. Thus, the keywords of mistake, ignorance and preconceived notion have been utilized along with serendipity.

It should be noted that while it is well known that Albert Einstein responded to the question from an interviewer saying that the accomplishments by the genius came from $1 \%$ of inspiration and $99 \%$ of perspiration, he later mentioned that my inventions and accomplishments never took place by accident, but continued hard and difficult work.

\section{References:}

Iijima, S.. (2010). Serendipity. Bungei Shunju, April, 84-86.

Ishikawa, A.. (2009). BI, knowledge management and crisis management 2\&3. Nikkan Kogyo Journal, June.

Ishikawa, A. \& Nakagawa, J.. (Eds.). (2009). Knowledge intelligence strategy. Tokyo: Tax and Accounting Association, 186-187.

Ishikawa, A. \& Tsujimoto, A.. (Eds.). (2008). Creative marketing for new product and new business development. New Jersey, London, Singapore: World Scientific Publishing Co.Pte.Ltd., 131-132, 138-139.

Singh, S.. (2006). Big bang, the origin of the universe. Shinchosha, 2, 206-209.

(Edited by Ruby and Chris) 